\title{
Influence of Railway Infrastructure on Train-To-Train Communications
}

\author{
Paul Unterhuber*, Michael Walter*, Thomas Kürner ${ }^{\dagger}$ \\ *Institute of Communications and Navigation, German Aerospace Center (DLR), Oberpfaffenhofen, 82234 Wessling, \\ Germany, paul.unterhuber@dlr.de \\ ${ }^{\dagger}$ Institute for Communications Technology, Technische Universität Braunschweig, 38106 Braunschweig, Germany
}

\begin{abstract}
The future of railway operation will rely on trainto-train (T2T) communications. Hence, we investigate the propagation mechanisms based on channel sounding measurements and derive channel models for T2T communications. For every wireless communication, the environment significantly influences the wireless propagation. For railway environments, especially regular installed objects along track like overhead line masts cause pronounced multipath components (MPCs).

In this paper, we analyze the MPCs from a T2T propagation measurement with two high speed trains. In detail, we extract the MPCs, identify the ones caused by overhead line masts and derive a distance-variant fading statistic for this kind of objects. Furthermore, we provide an outline on a T2T geometry-based stochastic channel model (GSCM) and how we incorporate the statistics of overhead line masts in the GSCM.
\end{abstract}

Index Terms-train-to-train, high speed train, propagation, GSCM, fading statistics.

\section{INTRODUCTION}

After decades of train-to-ground (T2G) based railway communication, future railway operation and railway applications like virtual coupled trains and fully autonomously driving trains will rely on T2T communications [1]. Since we have stated a lack of channel measurements and models in [2] in 2016, several projects focused on T2T propagation. Channel sounding measurements were presented for high speed trains and tracks in [3]; first results with respect to the power delay profile (PDP) and resulting path loss models were published in [4]. Another T2T channel sounding campaign for cargo trains in a shunting yard environment was presented in [5] and results on the PDP, path loss, delay spread and Doppler frequency spread were provided.

As we know from similar channel sounding measurements for transportation means as for car-to-car $(\mathrm{C} 2 \mathrm{C})$ or $\mathrm{T} 2 \mathrm{G}$ communication, the environment, the geometry of surrounding objects and the movement of the transmitter (Tx) and receiver $(\mathrm{Rx})$ have a significant influence on the wireless propagation. One example is the influence of overhead line masts on T2G communications as analyzed in [6]. Another example is the influence of cross bridges in a highway scenario for $\mathrm{C} 2 \mathrm{C}$ communication presented in [7]. In previous mentioned publications for T2T channel sounding as in [4], we could see pronounced MPCs in the PDP. Hence, interacting objects (IOs) have to be present along the track and cause MPCs, or may obstruct the line of sight (LOS) signal in curved track segments.
To the best of our knowledge, the particular influence of objects along the track on T2T communications has not been investigated so far. In [8] we presented an algorithm to localize and identify scatterer based on the delay and Doppler frequency of the MPC. Hence, we relate MPCs to IOs along the track and group the MPCs for similar objects. In this contribution we focus on overhead line masts and extract a distance-variant fading statistic for the scattering loss (SL) of overhead line masts.

The investigated geometry of the masts and the extracted statistics will be incorporated in a GSCM for T2T. An outline of the GSCM is provided.

\section{Measurement Campaign}

In 2016 we performed the world-wide first T2T channel sounder measurement campaign with two high speed trains. The measurements were conducted on a $205 \mathrm{~km}$ long high speed railway (HSR) track between Naples and Rome. The trains were driving on parallel tracks for safety reason. This gave us the freedom to perform departing, approaching and overtaking maneuvers with different velocities and in different environments. We installed various measurement equipment in two Trenitalia Frecciarossa ETR 500 [9] high speed trains [3].

\section{A. Measurement Equipment}

We performed C-band measurements with the DLR RUSK channel sounder in single-input single-output (SISO) mode. The channel sounder was set to a frequency of $f=5.2 \mathrm{GHz}$ with a bandwidth of $B=120 \mathrm{MHz}$ and recorded a discrete channel transfer function $H(t, f)$ with a snapshot rate of $t_{\mathrm{s}}=$ $1.024 \mathrm{~ms}$. The snapshot rate results in a maximum resolvable Doppler frequency of $\nu_{\max }= \pm 488.28 \mathrm{~Hz}$.

The maximum excess delay $t_{\mathrm{p}}$ was set to $12.8 \mu \mathrm{s}$ which results in a maximum path length of $d_{i, \max }=3.84 \mathrm{~km}$. The output power of the transmitter was limited to $27 \mathrm{dBm}$. Both trains were equipped with omni-directional antennas with an average gain of $6 \mathrm{dBi}$. The detailed settings are listed in Table I. The measurement equipment and setup was presented in more detail in [3]. 


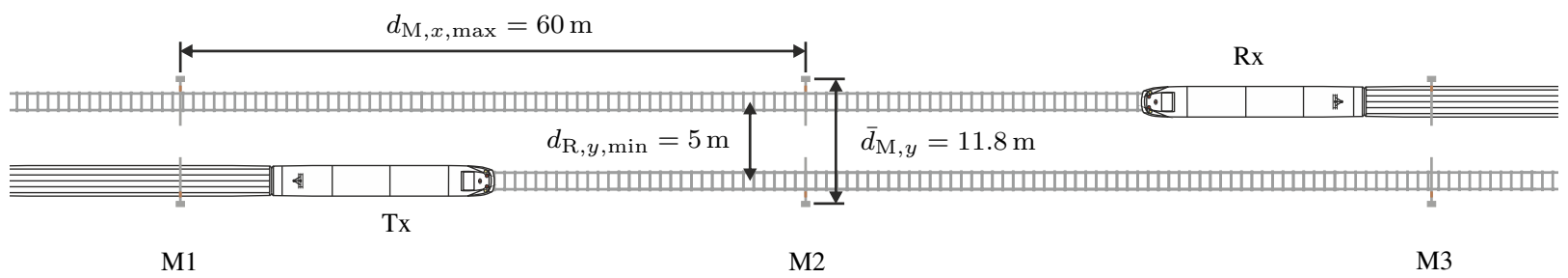

Fig. 1: Dimensions of the railway track and the overhead line masts along track.

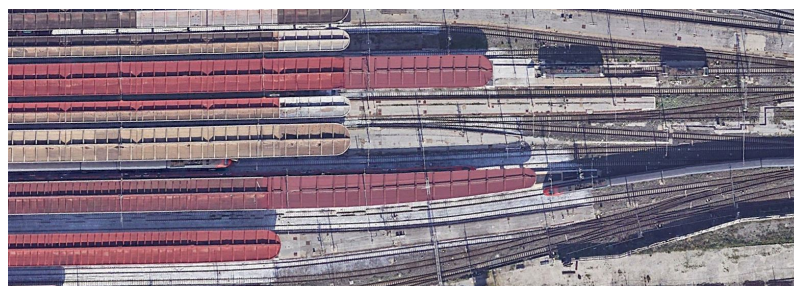

(a) Railway station.

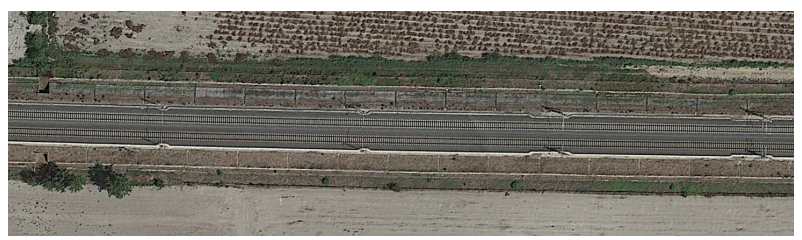

(b) Open field.

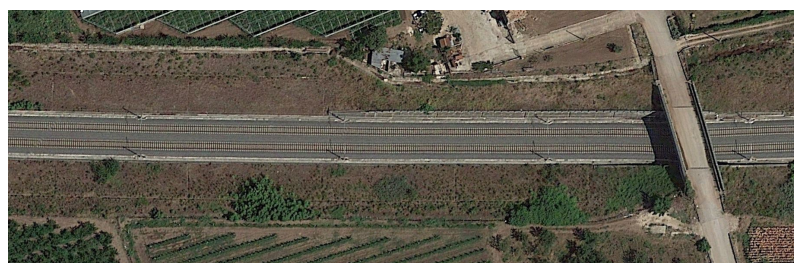

(c) Hilly terrain with cutting.

Fig. 2: Satellite images of the measurement environments. Image by Google Earth, Google 2020.

\begin{tabular}{|cc|c|}
\hline Parameter & & Value \\
\hline Frequency & $f$ & $5.2 \mathrm{GHz}$ \\
Bandwidth & $B$ & $120 \mathrm{MHz}$ \\
Snapshot rate & $t_{\mathrm{s}}$ & $1.024 \mathrm{~ms}$ \\
Excess delay & $t_{\mathrm{p}}$ & $12.8 \mu \mathrm{s}$ \\
Transmit power & $P_{\mathrm{T}}$ & $27 \mathrm{dBm}$ \\
\hline
\end{tabular}

TABLE I: Channel sounder settings.

\section{B. Measurement Environment}

The high speed track between Rome and Naples offers different environments on a to total length of $204.6 \mathrm{~km} .39 \mathrm{~km}$ run through tunnels and additional $39 \mathrm{~km}$ run on viaducts and bridges. The biggest share of the track runs through open field and hilly terrain with cutting environments [10].

\section{Track Architecture}

The track architecture of electrified HSRs is characterized by the rails and the overhead line system. The overhead line system with the catenary and the supporting masts remain over all environments and have a significant influence on the wireless propagation. The catenary runs above the track and the supporting masts are mounted to the left and right of the track in an average distance of $\bar{d}_{\mathrm{M}, y}=11.8 \mathrm{~m}$ between them. This results in a distance of $3.4 \mathrm{~m}$ from mast to track center. Along track, the masts appear in a maximum distance of $d_{\mathrm{M}, x, \max }=60 \mathrm{~m}$. The dimensions are shown in Fig. 1 .

The different environments are shown in Fig. 2; every environment influences the wireless propagation slightly different. In a railway station environment we observe a dense network of railway infrastructure like platforms, buildings, overhead systems, and signaling systems. In the open field environment the infrastructure reduces to the overhead line system and sparse signaling system components. In addition to the railway infrastructure cross bridges, close embankments or walls need to be considered for the hilly terrain with cutting environment.

\section{Measurement Results}

The T2T channel properties represented by the measured channel transfer function $H(t, f)$ are linked with a time stamp to global navigation satellite system (GNSS) position and velocity of the Tx and the Rx. T, $\boldsymbol{v}_{\mathrm{T}}$ and $\mathbf{R}, \boldsymbol{v}_{\mathrm{R}}$ represent the position and velocity of the Tx and $\mathrm{Rx}$, respectively.

\section{A. Stochastic Channel Description}

By applying an inverse Fourier transformation in the frequency domain and a Fourier transformation in the time domain, the measured time-variant $H(t, f)$ can be represented by the time-variant spreading function as

$$
s(\nu, \tau ; t, f)=\frac{1}{\sqrt{I J}} \sum_{t=0}^{J-1} \sum_{f=0}^{I-1} H(t, f) \mathrm{w}(t, f) \mathrm{e}^{-j 2 \pi(\nu t-\tau f)} .
$$

$\mathrm{w}(t, f)$ represents the time-frequency dependent window function. $I$ and $J$ represent the time and frequency stationarity windows. We assume uncorrelated scattering within the measurement bandwidth and set $I=1537$ bins which represent $B=120 \mathrm{MHz}$. By assuming a maximum relative velocity of $\Delta v_{\max }=50 \mathrm{~km} / \mathrm{h}$ we can estimate a stationarity window as

$$
t_{\text {stat }}=\frac{10 \lambda}{\left|\Delta v_{\max }\right|}=41.5 \mathrm{~ms} \text {. }
$$




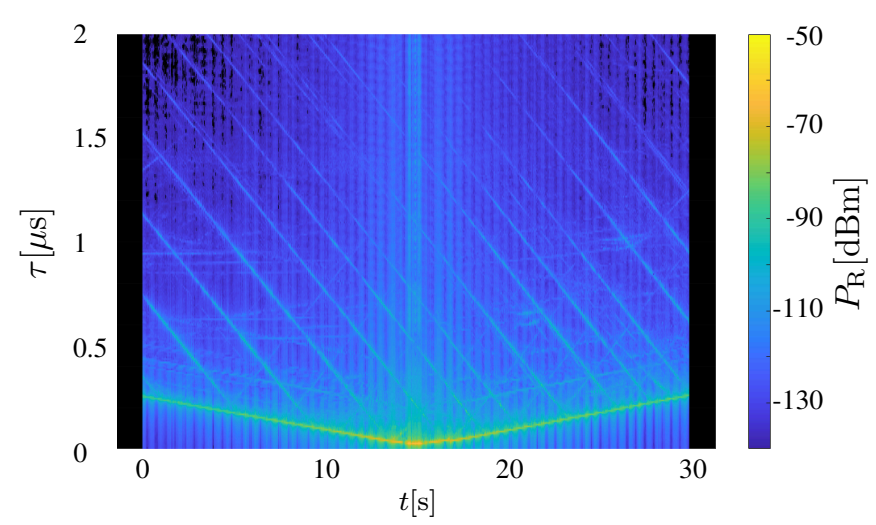

Fig. 3: PDP of the measurement data of an overtake maneuver in open field environment.

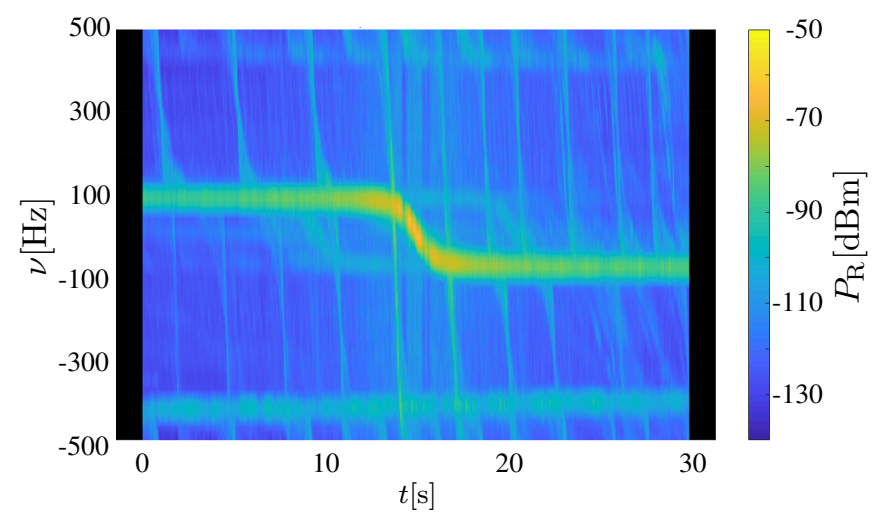

Fig. 4: DSD of the measurement data of an overtake maneuver in open field environment.

We set the a window length of $J=64$ to meet the stationarity time constraints and to resolve the Doppler frequency sufficiently. As we assume uncorrelated scattering, the timefrequency-variant scattering function $s(\nu, \tau ; t, f)$ can be reduced to a time-variant function $s(\nu, \tau ; t)$.

The time-variant PDP is derived as

$$
P_{h}(\tau ; t)=\frac{1}{J} \sum_{\nu=-J / 2}^{J / 2-1}|s(\nu, \tau ; t)|^{2}
$$

and the time-variant Doppler spectral density (DSD) as

$$
P_{S}(\nu ; t)=\frac{1}{I} \sum_{\tau=0}^{I-1}|s(\nu, \tau ; t)|^{2} .
$$

As an example, the PDP and DSD of an overtake maneuver in open field environment is shown in Fig. 3 and Fig. 4. We clearly see the LOS path and the MPCs in both figures.

\section{B. Parameter Estimation}

The Kalman enhanced super resolution tracking (KEST) algorithm was used to estimate the MPC parameters. The algorithm was introduced by Jost in [11] and an adaptation

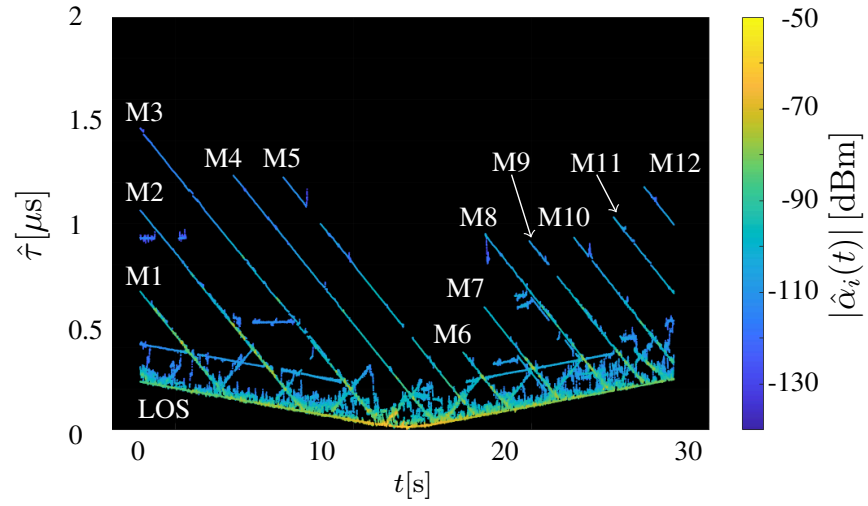

Fig. 5: PDP of the LOS path and the tracked MPCs by the KEST algorithm.

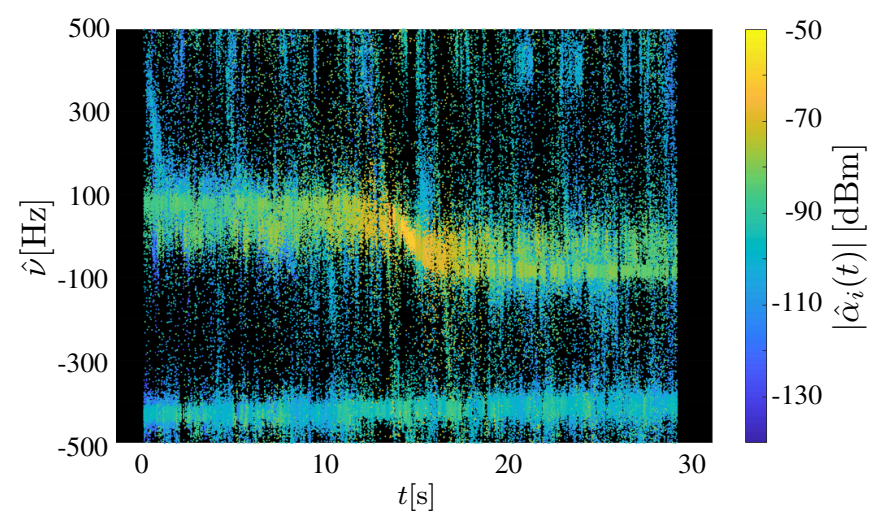

Fig. 6: DSD of the LOS path and the tracked MPCs by the KEST algorithm.

to moving Tx and moving Rx was presented in [12]. Briefly speaking, KEST is designed in two stages with an inner stage based on the space-alternating generalized expectationmaximization (SAGE) algorithm introduced in [13] and an outer stage based on Kalman filters.

The KEST algorithm provides an estimate of the channel impulse response similar to a sum of weighted and distorted pulses $\chi_{i}[14]$ as

$$
\hat{h}(t, \tau)=\sum_{i=0}^{N(t)-1} \hat{\alpha}_{i}(t) \chi_{i}\left(\tau-\hat{\tau}_{i}(t)\right) .
$$

We count a total amount of $N$ MPCs. For each time instant $t$ of the $i^{\text {th }}$ MPC the weight is represented by the estimated complex amplitude $\hat{\alpha}_{i}(t)=\left|\hat{\alpha}_{i}(t)\right| e^{j \hat{\varphi}_{i}(t)}$ and the distortion by the delay $\hat{\tau}_{i}(t)$. Similar to the pure measurement data, we also see the estimated and tracked LOS and the MPCs in Fig. 5 in the same domain as for the PDP representation and in Fig. 6 for the DSD representation.

The absolute amplitude $\left|\hat{\alpha}_{i}(t)\right|$ is used for the fading statistics; the phase information $\hat{\varphi}_{i}(t)$ of the amplitude is used to estimate the Doppler frequency of the $i^{\text {th }}$ MPC as

$$
\hat{\nu}_{i}(t)=-\frac{1}{2 \pi t_{\mathrm{s}}} \frac{\partial \hat{\varphi}_{i}(t)}{\partial t} \text {. }
$$




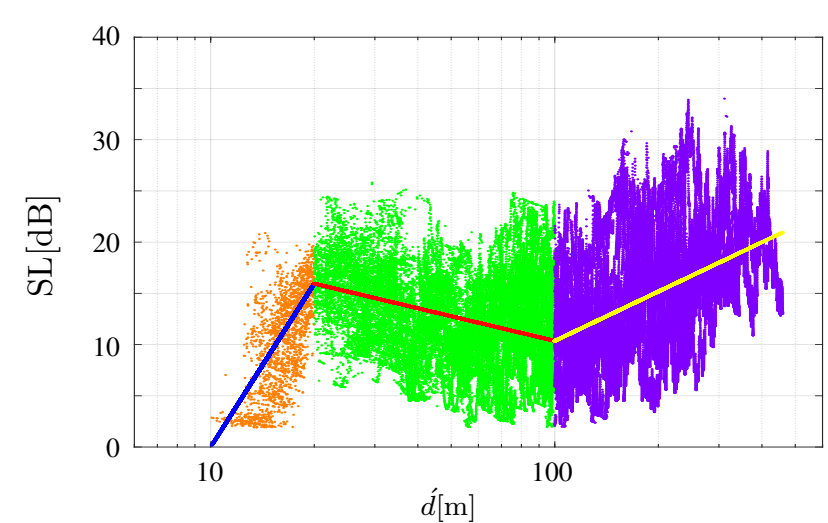

Fig. 7: SL for overhead line masts indicated as orange, green and purple dots for three different distance parts. The related fitting is represented by the blue, red and yellow line.

\section{Scatterer Identification}

The estimated delay $\hat{\tau}_{i}(t)$ and derived Doppler frequency $\hat{\nu}_{i}(t)$ are used jointly to estimate the scatterer location and identify the underlying IO. The algorithm was introduced in [15] for a fixed $\mathrm{Tx}$ and moving $\mathrm{Rx}$ configuration. The extension to moving Tx and moving Rx was presented in [8].

\section{FADING STATISTICS FOR OVERHEAD LINE MASTS}

The highlighted MPCs M1 to M12 in Fig. 5 were identified as MPCs caused by overhead line masts. We normalize the absolute amplitude $\left|\hat{\alpha}_{i}(t)\right|$ by the Tx power $P_{\mathrm{T}}$ and all gains and losses of the measurement chain $A_{\text {sys }}$ in $\mathrm{dB}$. In this way we receive an estimated path loss $\mathrm{PL}_{i}(t)$ for each MPC $i$. By subtracting the free-space path loss (FSPL) representing the path length of the MPC $d_{i}(t)$ we receive the SL caused by the scattering on the surface of the overhead line mast as

$$
\begin{aligned}
\mathrm{SL}_{i}(t)= & \mathrm{PL}_{i}(t)-F S P L\left(d_{i}(t)\right) \\
= & -20 \log _{10}\left(\left|\hat{\alpha}_{i}(t)\right|\right) \\
& +A_{\text {sys }} \\
& -20 \log _{10}\left(\frac{4 \pi d_{i}(t) f}{c}\right) .
\end{aligned}
$$

We split the analysis in three parts based on the geometry of the Tx, the Rx and the IO, e.g. the mast is

- in front of Tx and Rx,

- in between of Tx and Rx,

- or behind the Tx and Rx.

In case of the second setup, the MPC fall in the same delay bin as the LOS and cause fading on the LOS signal. The analysis of the LOS fading is out of scope of this publication.

For the first and the third geometry, we normalize the estimated delay $\tau_{i}(t)$ of each MPC $i$ to the LOS delay $\tau_{\text {LOS }}(t)$ at time instant $t$. In this way, we shrink the geometry as Tx and $\mathrm{Rx}$ would be on the same position along track. Hence, a common statistic of the SL can be derived based on the residual path length $d^{\prime}(t)=d_{i}(t)-d_{\mathrm{LOS}}(t)$. As the highlighted

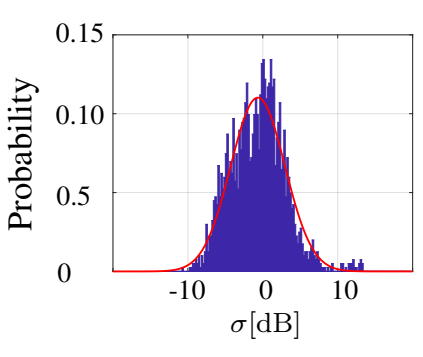

(a)

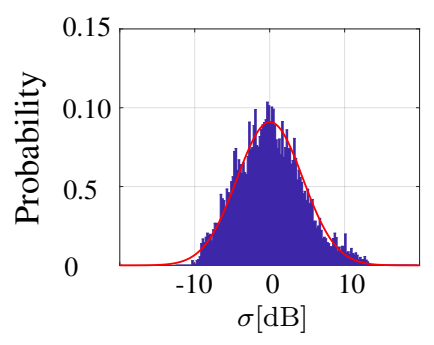

(b)



(c)

Fig. 8: Log-normal distribution for overhead line masts for three segments of the SL model: (a) short distances, (b) mid distances (c) long distances. The blue histogram indicates the estimated SL and the red line the fitted Gaussian distribution.

estimated MPCs relate to masts in front of the trains, we focus in this publication on the first geometry. Nevertheless, due to the symmetry of the geometry for overhead line masts, we assume a similar behavior for the first and the third geometry.

In Fig. 7 we show the derived SL for all estimated MPCs in relation to $d$. The residual path length is split in three parts: Short distances with $\dot{d}=[10,20] \mathrm{m}$, mid distances with $d=$ $(20,100] \mathrm{m}$ and far distances with $d \dot{d}>100 \mathrm{~m}$. In all three parts we fitted a log-normal function

$$
\mathrm{S} \dot{L}=\mathrm{S} L\left(\dot{d}_{0}\right)+10 m \log _{10}\left(\frac{\dot{d}}{\dot{d}_{0}}\right)+X_{\sigma},
$$

with a SL exponent $m$, at a reference distance $d_{0}$ and Gaussian random variable $X_{\sigma}=\mathcal{N}\left(0, \sigma^{2}\right)$.

In the near region, indicated by the orange point cloud, we see a strong increase of the SL from 2 to $20 \mathrm{~dB}$. At the reference distance we set the SL $\left(\dot{d}_{0}=10 \mathrm{~m}\right)=0 \mathrm{~dB}$. The corresponding fit is indicated as blue line with an exponent of $m=5.3$ and a standard deviation of $\sigma=3.6 \mathrm{~dB}$. In the mid region from $20 \mathrm{~m}$ to $100 \mathrm{~m}$, indicated as green dots, the SL slightly decreases and is fitted by $m=-0.8$ and $\sigma=4.4 \mathrm{~dB}$, starting at $\mathrm{SL}\left(\hat{d}_{0}=20 \mathrm{~m}\right)=15.95 \mathrm{~dB}$ plotted as red line in Fig. 7. The purple indicated SL for larger distances above $100 \mathrm{~m}$ show strong variations with an increasing trend. The fitted log-normal function shown as yellow line results in a exponent $m=1.6$ and a standard deviation $\sigma=5.0 \mathrm{~dB}$ with ŚL $\left(\dot{d}_{0}=100 \mathrm{~m}\right)=10.36 \mathrm{~dB}$. All parameters are listed in Table II.

The overhead line masts along the measurement track were lattice masts. Depending on the incoming and outgoing angle 
TABLE II: SL fitting parameters.

\begin{tabular}{|c|c|c|c|c|}
\hline Distance & $d_{0}[\mathrm{~m}]$ & $\mathrm{SL}\left(\hat{d}_{0}\right)[\mathrm{dB}]$ & $m$ & $\sigma[\mathrm{dB}]$ \\
\hline short & 10 & 0 & 5.3 & 3.6 \\
mid & 20 & 15.95 & -0.8 & 4.4 \\
far & 100 & 10.36 & 1.6 & 5.0 \\
\hline
\end{tabular}

of the measurement signal the SL changes around the quadratic outline of the mast. For $d$ around $10 \mathrm{~m}$, the signal is scattered on a rather large surface facing parallel to the track. By increasing the path length, the scatter point moves toward the edge of the mast and SL increases significantly. Up from $d=20 \mathrm{~m}$ the signal is scattered on the perpendicular oriented phase of the mast. Hence, the signal is scattered on a large surface and $S^{\prime} L$ indicated as red line in Fig. 7 decreases slightly. For $d \dot{d}>100 \mathrm{~m}$ the increasing exponent $m$ reflects the increasing path loss for larger distances in comparison to FSPL with an path loss exponent of $n=2$.

\section{T2T GSCM OUTLINE}

The T2T GSCM will consist of a LOS part and of a MPC part based on the underlying geometry of the environment. The simplest environment, the open field environment, consists of overhead line masts only. The position of overhead line masts along the HSRs have been analyzed and statistics for the distance to the track and along the track have been derived. Based on these statistics, the masts will be placed in the model randomly.

The LOS part will reflect the distance between and the movement of the Tx and Rx. The path loss and delay will be calculated based on the distance, the Doppler frequency will represent the relative movement of the Tx and Rx. The movement and distance will be arbitrary.

The MPC part will be split in two parts. The first part will consist of a geometry and movement dependent path loss, delay and Doppler frequency estimation. Based on the geometry between the Tx, the IO and the Rx, the path loss and delay will be estimated similar to the LOS part. The relative movement between the Tx, the IO and the Rx will be taken into account with a Doppler frequency estimation. Second, an IO related stochastic propagation parameter will be introduced to consider the attenuation due to lossy reflection and scattering. Hence, the derived SL parameters for overhead line masts are one fundamental step towards the T2T GSCM.

\section{CONCLUSION}

It is undisputed that the environment significantly influences wireless propagation. Our channel sounding measurement data from a T2T measurement campaign shows pronounced MPCs caused by the railway environment. We extracted and tracked the complex amplitude and delay of each MPC over time with the KEST algorithm. Furthermore, we used the channel estimation to identify the underlying IO of each MPC. In this way, we could allocate the estimated channel parameters to real objects. In open field environment, most MPCs could be identified as MPCs caused by overhead line masts.
Based on our measurements and the estimated channel parameters we extracted time-variant SL coefficients for overhead line masts. We analyzed the results in relation to the distance and fitted a log-normal function for three different regions. The statistical SL description will be a major input for a future T2T GSCM. An outline of the GSCM was proved as well with details on the LOS and MPC signal modeling approach.

\section{REFERENCES}

[1] J. Moreno García-Loygorri, J. Goikoetxea, E. Echeverria, A. Arriola, I. Val, S. Sand, P. Unterhuber, and F. del Rio, "The wireless train communication network: Roll2rail vision," IEEE Vehicular Technology Magazine, vol. 13, no. 3, pp. 135-143, 092018.

[2] P. Unterhuber, S. Pfletschinger, S. Sand, M. Soliman, T. Jost, A. Arriola, I. naki Val, C. Cruces, Moreno García-Loygorri, J. GarcíaNieto, C. Rodríguez, M. Berbineau, E. Echeverría, and I. Baz, "A Survey of Channel Measurements and Models for Current and Future Railway Communication Systems," Mobile Information Systems, vol. 2016, pp. 1-14, June 2016. [Online]. Available: http://dx.doi.org/10.1155/2016/7308604

[3] P. Unterhuber, S. Sand, M. Soliman, B. Siebler, A. Lehner, T. Strang, M. d'Atri, F. Tavano, and D. Gera, "Wide Band Propagation in Trainto-Train Scenarios - Measurement Campaign and First Results," in 2017 11th European Conference on Antennas and Propagation (EuCAP), 03 2017, pp. 3356-3360.

[4] P. Unterhuber, I. Rashdan, M. Walter, and T. Kürner, "Path loss models and large scale fading statistics for c-band train-to-train communication," in 14th European Conference on Antennas and Propagation (EuCAP 2020), 04 2020, pp. 1-5.

[5] S. Zelenbaba, L. W. Mayer, E. Mozo, F. Wirth, R. Hladik, A. A. Gómez, L. Bernadó, M. Schiefer, and T. Zemen, "Characterization of timevariant wireless channels in railway communication scenarios," in 2019 IEEE 2nd 5G World Forum (5GWF), 2019, pp. 536-541.

[6] L. Zhou, Z. Yang, F. Luan, A. Molisch, F. Tufvesson, and S. Zhou, "Dynamic channel model with overhead line poles for high-speed railway communications," IEEE Antennas and Wireless Propagation Letters, vol. 17, no. 5, pp. 903-906, 052018.

[7] A. Paier, L. Bernado, J. Karedal, O. Klemp, and A. Kwoczek, "Overview of vehicle-to-vehicle radio channel measurements for collision avoidance applications," in 2010 IEEE 71st Vehicular Technology Conference, 05 2010, pp. 1-5.

[8] P. Unterhuber, M. Walter, N. Schneckenburger, and T. Kürner, "Joint delay and doppler frequency estimation for scatterer localization in railway environments," in 2019 13th European Conference on Antennas and Propagation (EuCAP), 03 2019, pp. 1-5.

[9] Trenitalia, "ETR 500," 2020. [Online]. Available: https://www.trenitalia com/de/frecce/frecciarossa_etr500.html

[10] Wikipedia, "Rome-Naples high-speed railway," 2018. [Online]. Available: https://en.wikipedia.org/wiki/Rome\%E2\%80\%93Naples_ high-speed_railway

[11] T. Jost, W. Wang, U.-C. Fiebig, and F. Perez-Fontan, "Detection and Tracking of Mobile Propagation Channel Paths," IEEE Transactions on Antennas and Propagation, vol. 60, no. 10, pp. 4875-4883, 102012.

[12] P. Unterhuber, T. Jost, W. Wang, and T. Kürner, "Measurement based spatial characteristics of mpcs in train-to-train propagation," in 12th European Conference on Antennas and Propagation (EuCAP 2018), 04 2018, pp. 1-5.

[13] B. H. Fleury, M. Tschudin, R. Heddergott, D. Dahlhaus, and K. I. Pedersen, "Channel Parameter Estimation in Mobile Radio Environments Using the SAGE Algorithm," IEEE Journal on Selected Areas in Communications, vol. 17, no. 3, pp. 434-450, 031999.

[14] A. Molisch, "Ultra-Wide-Band Propagation Channels," Proceedings of the IEEE, vol. 97, no. 2, pp. 353-371, 022009.

[15] N. Schneckenburger, T. Jost, D. Shutin, M. Walter, G. del Galdo, and U.-C. Fiebig, "Reflector localization for geometrical modeling the airground channel," IEEE Transactions on Vehicular Technology, pp. 1-1, 2018. 